\title{
The coolest extremely low-mass white dwarfs
}

\author{
Leila M. Calcaferro ${ }^{1,2}$, Leandro G. Althaus ${ }^{1,2}$, and Alejandro H. Córsico ${ }^{1,2}$ \\ ${ }^{1}$ Grupo de Evolución Estelar y Pulsaciones, Facultad de Ciencias Astronómicas y Geofísicas, Universidad Nacional de La Plata, \\ Paseo del Bosque s/n, 1900 La Plata, Argentina \\ e-mail: lcalcaferro@fcaglp.unlp.edu.ar; althaus@fcaglp.unlp.edu.ar; acorsico@fcaglp.unlp.edu.ar \\ 2 Instituto de Astrofísica La Plata, CONICET-UNLP, Paseo del Bosque s/n, 1900 La Plata, Argentina
}

Received 26 December 2017 / Accepted 19 February 2018

\begin{abstract}
Context. Extremely low-mass white dwarf (ELM WD; $M_{\star} \lesssim 0.18-0.20 M_{\odot}$ ) stars are thought to be formed in binary systems via stable or unstable mass transfer. Although stable mass transfer predicts the formation of ELM WDs with thick hydrogen (H) envelopes that are characterized by dominant residual nuclear burning along the cooling branch, the formation of ELM WDs with thinner $\mathrm{H}$ envelopes from unstable mass loss cannot be discarded.

Aims. We compute new evolutionary sequences for helium $(\mathrm{He})$ core WD stars with thin $\mathrm{H}$ envelopes with the main aim of assessing the lowest $T_{\text {eff }}$ that could be reached by this type of stars.

Methods. We generate a new grid of evolutionary sequences of He-core WD stars with thin $\mathrm{H}$ envelopes in the mass range from 0.1554 to $0.2025 M_{\odot}$, and assess the changes in both the cooling times and surface gravity induced by a reduction of the $\mathrm{H}$ envelope. We also determine, taking into account the predictions of progenitor evolution, the lowest $T_{\text {eff }}$ reached by the resulting ELM WDs.

Results. We find that a slight reduction in the $\mathrm{H}$ envelope yields a significant increase in the cooling rate of ELM WDs. Because of this, ELM WDs with thin $\mathrm{H}$ envelopes could cool down to $\sim 2500 \mathrm{~K}$, in contrast to their canonical counterparts that cool down to $\sim 7000 \mathrm{~K}$. In addition, we find that a reduction of the thickness of the H envelope markedly increases the surface gravity $(g)$ of these stars.

Conclusions. If ELM WDs are formed with thin $\mathrm{H}$ envelopes, they could be detected at very low $T_{\text {eff }}$. The detection of such cool ELM WDs would be indicative that they were formed with thin $\mathrm{H}$ envelopes, thus opening the possibility of placing constraints on the possible mechanisms of formation of this type of star. Last but not least, the increase in $g$ due to the reduction of the $\mathrm{H}$ envelope leads to consequences in the spectroscopic determinations of these stars.
\end{abstract}

Key words. stars: evolution - stars: interiors - stars: individual: ELM WD - white dwarfs

\section{Introduction}

White dwarf (WD) stars constitute the most common fate in stellar evolution. Indeed, more than $90 \%$ of all stars born in the galaxy will ultimately evolve into WDs, earth-sized, electron degenerate objects. These stars play a central and unique role in our understanding of the formation and evolution of stars, our galaxy itself, and planetary systems. In addition, they constitute valuable laboratories of extreme physics. Their collective properties allow us to infer valuable information about the star formation history of the Solar neighborhood, asymptotic giant branch (AGB) mass loss, and to study the properties of various stellar populations; see Fontaine \& Brassard (2008), Winget \& Kepler (2008), and Althaus et al. (2010) for reviews. In particular, WDs are used as accurate age indicators for a wide variety of Galactic populations, including the disk, and open and globular clusters; see Winget et al. (2009), García-Berro et al. (2010), Jeffery et al. (2011), Bono et al. (2013), Hansen et al. (2013) and Torres et al. (2015) for some recent applications.

The WD mass distribution comprises a population of lowmass remnants, most of them expected to have a He core. These stars are thought to be the result of strong mass-loss episodes in interactive binary systems before the He flash during the red giant branch (RGB)-phase of low-mass stars; see Sarna et al. (2000), Althaus et al. (2013), Istrate et al. (2016), and references therein. Specifically, this interactive binary evolutionary scenario is thought to be the most plausible origin for the so-called extremely low-mass (ELM) WDs, which have masses below $\sim 0.18-0.20 M_{\odot}{ }^{1}$.

In recent years, the number of observed low-mass WDs, including ELM WDs, has increased considerably due to the result of many ELM surveys, and the SPY and WASP surveys (e.g., Koester et al. 2009; Brown et al. 2010; Maxted et al. 2011; Kilic et al. 2015; Gianninas et al. 2015). The evolution of He-core WDs resulting from binary evolution has recently been studied in detail by Althaus et al. (2013) and Istrate et al. (2016). In addition, the detection of pulsation $g$ modes (gravity modes) in some of these stars (Hermes et al. 2012, 2013b,a; Kilic et al. 2015; Bell et al. 2015, 2017) has given rise to a new class of variable WDs, the ELM variables (ELMVs). These pulsating low-mass WDs provide a unique chance to probe the interiors of these stars and possibly to test their formation scenarios by employing the tools of asteroseismology.

The accepted mechanism for the formation of low-mass He-core WDs is either through unstable mass loss via commonenvelope episodes or stable mass loss via Roche-lobe overflow in close binary systems (see for a recent discussion Istrate et al. 2016). In particular, existing evolutionary tracks for ELM WDs are derived from progenitor stars that have experienced stable mass transfer; see Althaus et al. (2013); Istrate et al. (2016) and references therein. All of these studies predict for these stars

\footnotetext{
1 In this paper we consider ELM WDs to be those He-core WDs for which no CNO H shell flashes are expected during the cooling phase.
} 
the occurrence of thick $\mathrm{H}$ envelopes that sustain residual stable $\mathrm{H}$ burning, thus yielding extremely long cooling ages even at high effective temperatures. In particular, these studies show that no ELM (those which did not experience CNO $\mathrm{H}$ flashes) is expected to have cooled below $\sim 7000 \mathrm{~K}$.

However, the formation of low-mass He-core WDs with thin $\mathrm{H}$ envelopes unable to sustain residual $\mathrm{H}$ burning cannot be discarded. Such WDs could result from common-envelope evolution of close binary systems (see Nandez \& Ivanova (2016), Ivanova \& Nandez (2016), Clayton et al. (2017) for recent calculations) or from the loss of the envelope of a RGB star induced by an inspiralling giant planet (Nelemans \& Tauris 1998; De Marco \& Soker 2002; Sabach \& Soker 2018). These studies suggest that most of the envelope of an RGB star could indeed be lost in these episodes. In particular, Clayton et al. (2017) found that the envelope of their low-mass RGB models becomes dynamically unstable, resulting in the entire envelope of the star being removed over the duration of the slow spiral-in phase. However, it should be mentioned that the prediction of such channels for the formation of ELM WDs is more uncertain, because of the large binding energy of the donor's envelope in this case (Sun \& Arras 2018). On the observational side, the existence of a population of low-mass He-core WDs with thin $\mathrm{H}$ envelopes in NGC 6397 is not discarded; see Strickler et al. (2009). Finally, in an entirely different context, the formation of ELM WDs with thin $\mathrm{H}$ envelope could result from the irradiation of the pulsar companion, leading to a fast cooling of the ELM (van den Heuvel \& van Paradijs 1988; Ergma et al. 2001).

In view of these considerations, we cannot entirely rule out the existence of ELM WDs with thin H envelopes. To explore the impact of this possible outcome of binary interaction on observational expectations of ELM WDs, we present in this paper new evolutionary sequences for ELM WDs formed with a thin $\mathrm{H}$ envelope. We show that in this case the cooling times of such WDs turn out to be much shorter than the cooling times of their counterparts with thick (canonical) $\mathrm{H}$ envelopes that suggest no ELM WD is expected to be observed below $\sim 7000 \mathrm{~K}$, in agreement with the current observational status. According to our calculations, however, if ELM WDs were born with thin $\mathrm{H}$ envelope (for instance, due to unstable mass transfer) they could be observed at much lower effective temperatures (down to $2500 \mathrm{~K}$ ). The detection of ELM WDs at low effective temperatures would be indicative that such WDs were formed with a thin $\mathrm{H}$ envelope, a fact that could shed light on the nature of mass loss that leads to the formation of ELM WDs. Although the detection of such cool ELM WDs is difficult, due to the spectroscopic technique becoming inaccurate below $7000 \mathrm{~K}$ where the Balmer lines become weaker and then disappear below $5000 \mathrm{~K}$, parallax measurements from Gaia represent a promising avenue that would help to identify such cool ELM WDs.

This paper is organized as follows. A brief description of the stellar models and the numerical code employed is provided in Sect. 2. In Sect. 3 we study the cooling times for some selected ELM WD sequences with thin and thick $\mathrm{H}$ envelopes. Subsequently, we describe the considerations that we take into account to find the lowest effective temperature that could be reached by ELM WD stars considering different possible progenitors and we show the results obtained. Finally, in Sect. 4 we summarize the main findings of this work.

\section{Evolutionary models}

This work is based on fully evolutionary models of low-mass Hecore WDs generated with the LPCODE stellar evolution code. This code computes in detail the complete evolutionary stages that lead to the WD formation, allowing the study of the WD evolution consistently with the predictions of the evolutionary history of progenitors. Details of the LPCODE can be found in Althaus et al. $(2005,2009,2013,2015)$ and references therein. Here, we briefly mention the ingredients employed that are relevant for our analysis of low-mass He-core WDs (see Althaus et al. 2013, for details). The standard mixing length theory (MLT) for convection in the ML2 prescription is used (see Tassoul et al. 1990, for its definition). We assume the metallicity of the progenitor stars to be $Z=0.01$. For the WD regime, we consider the radiative opacities for arbitrary metallicity in the range of 0 to 0.1 from the OPAL project (Iglesias \& Rogers 1996). Conductive opacities are those of Cassisi et al. (2007). For the main sequence evolution, we consider the equation of state from OPAL for $\mathrm{H}$ - and He-rich compositions. Neutrino emission rates for pair, photo, and bremsstrahlung processes are those of Itoh et al. (1996), and for plasma processes, those of Haft et al. (1994). For the WD regime we have employed an updated version of the equation of state of Magni \& Mazzitelli (1979). The nuclear network takes into account 16 elements and 34 thermonuclear reaction rates, most of them corresponding to pp-chains and $\mathrm{CNO}$ bicycle needed for this work. We also consider time-dependent diffusion due to gravitational settling and chemical and thermal diffusion of nuclear species following the multicomponent gas treatment of Burgers (1969). We have computed abundance changes according to element diffusion, nuclear reactions, and convective mixing, a treatment that represents a very significant aspect in evaluating the importance of residual nuclear burning during the cooling stage of low-mass WDs.

Realistic configurations for our initial low-mass He-core WD models were taken from binary evolutionary calculations (Althaus et al. 2013). There, binary evolution was assumed to be fully nonconservative, and the losses of angular momentum due to mass loss, gravitational wave radiation, and magnetic braking were considered. Binary configurations assumed in Althaus et al. (2013) consist of an evolving main sequence low-mass component (donor star) of initially $1 M_{\odot}$ and a $1.4 M_{\odot}$ neutron star companion as the other component. Initial He-core WD models with stellar masses ranging from 0.1554 to $0.4352 M_{\odot}$ characterized by thick $\mathrm{H}$ envelopes were derived from stable mass loss via Roche-lobe overflow; see Althaus et al. (2013) for details. The evolution of these models was computed down to the range of luminosities of cool WDs, including the stages of multiple thermonuclear $\mathrm{CNO}$ flashes at the beginning of the cooling branch.

Initial ELM WD models with thin $\mathrm{H}$ envelopes were generated from those computed in Althaus et al. (2013), as mentioned above, by artificially reducing the thickness of the $\mathrm{H}$ envelope at high luminosities, during the pre-white dwarf evolutionary stages. This ensures that all the transitory effects associated with this artificial procedure have already ceased by the time the resulting remnants reach the cooling branch. In order to achieve different thicknesses of the $\mathrm{H}$ envelope, for each sequence characterized by a given $M_{\star}$ and a thick (canonical) value of $M_{\mathrm{H}}$, as predicted by the full computation of the pre-WD evolution, we simply replaced ${ }^{1} \mathrm{H}$ by ${ }^{4} \mathrm{He}$ from a given mesh point to obtain the desired $\mathrm{H}$ envelope mass. Ongoing element diffusion smoothes the chemical profile at the $\mathrm{H} / \mathrm{He}$ chemical transition region before the remnant settles onto the cooling branch. With the aim of exploring not only the impact of the $\mathrm{H}$ envelope on the cooling time, for which only a small reduction in the envelope thickness is required, but also on the surface gravity, we have generated additional sequences with $\mathrm{H}$ envelope several orders 


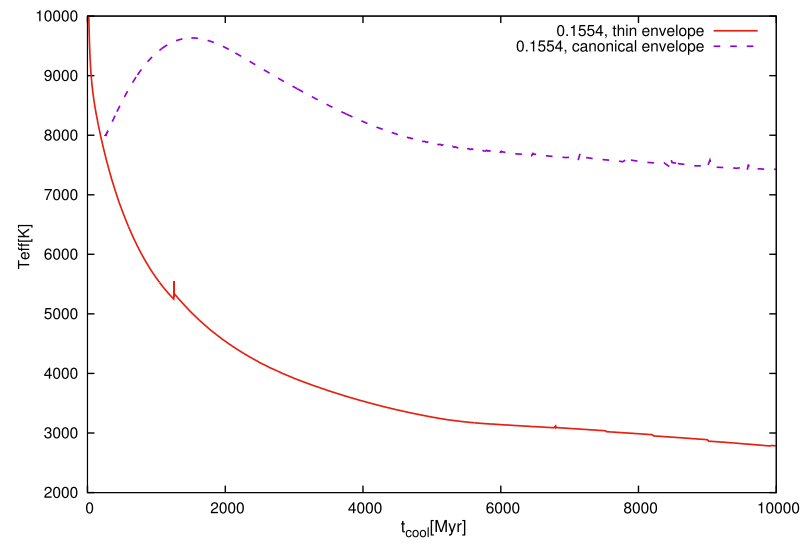

Fig. 1. Cooling curves for the ELM WDs with $M_{\star}=0.1554 M_{\odot}$ with both canonical and thin $\mathrm{H}$ envelopes.

of magnitude thinner than the canonical value. We have also generated two evolutionary sequences with 0.130 and $0.140 M_{\odot}$ with thin $\mathrm{H}$ envelopes to cover the domain of low surface gravities of our grid. This was done by artificially scaling the total mass of the $0.1554 M_{\odot}$ white dwarf model.

\section{Searching for ELMs with the lowest effective temperature}

We begin by examining the impact of the $\mathrm{H}$ envelope mass on the cooling times of the ELM WDs. In this sense, we find that a small reduction in the $\mathrm{H}$ envelope, by a factor of two to three, is enough to turn off the $\mathrm{H}$ burning, thus leading to a fast evolution at advanced stages (see Althaus et al. 2001, for a similar result). This is illustrated in Figs. 1 and 2, which display the cooling times as a function of the effective temperature for the sequences with 0.1554 and $0.1822 M_{\odot}$, respectively. We stress that these sequences did not experience CNO flashes on the cooling branch. Cooling times are shown for both canonical and thin $\mathrm{H}$ envelopes. The figures show the fast cooling that characterizes sequences with thin $\mathrm{H}$ envelopes, as compared with their counterparts with canonical envelopes powered by residual $\mathrm{H}$ burning. In particular, for the $M_{\star}=0.1554 M_{\odot}$ canonical sequence, evolution takes roughly $8700 \mathrm{Myr}$ to cool down to an effective temperature of $7500 \mathrm{~K}$, in contrast with the $290 \mathrm{Myr}$ required by the thin envelope sequence. More specific information is provided by Table 1 , which tabulates the cooling time, $t_{\text {cool }}$, along with $\log (g)$ for some selected $0.1554,0.1822$, and $0.2025 M_{\odot}$ models having canonical (with $M_{\mathrm{H}}=2.0 \times 10^{-2}, 3.6 \times 10^{-3}$ and $3.7 \times 10^{-3} M_{\star}$ for the $0.1554,0.1822$, and $0.2025 M_{\odot}$ sequences, respectively) and thin $\mathrm{H}$ envelopes $\left(M_{\mathrm{H}} \sim 10^{-5} M_{\star}\right.$ for all the sequences). We mention that the $0.2025 M_{\odot}$ sequence experiences $\mathrm{CNO}$ flashes during the first cooling branch, with the consequent result being that the $\mathrm{H}$ envelope and $\mathrm{H}$ burning on the final cooling branch are reduced. This explains the short cooling age of this sequence also in the case of canonical envelope. It is clear that residual nuclear burning is responsible for the extremely large cooling times that would need ELM WDs to reach low effective temperatures, and more importantly, that a small reduction of the $\mathrm{H}$ envelope is enough to extinguish this nuclear burning source, and thus cause a fast evolution of the ELM WD.

The short cooling times that characterize the sequences with thin $\mathrm{H}$ envelopes open the possibility of detecting ELM WDs at very low $T_{\text {eff }}$. To quantify this, we assume an age of the Galactic

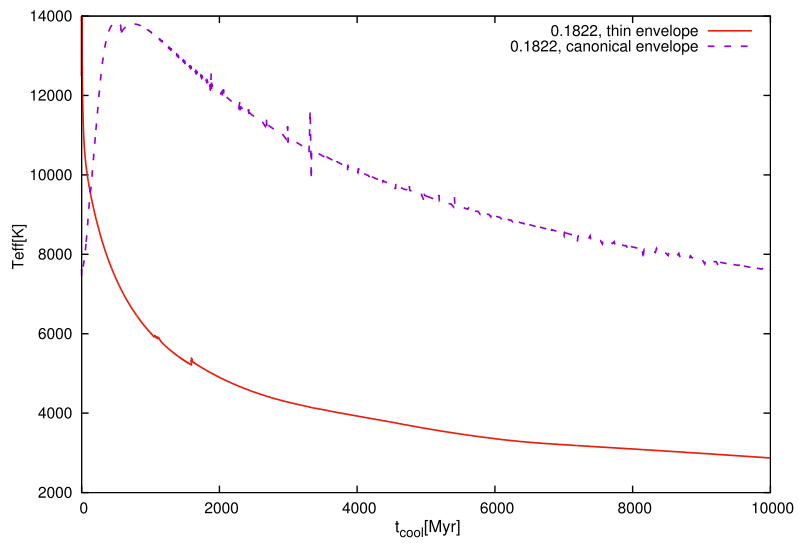

Fig. 2. As in Fig. 1 but for the evolutionary sequences with $M_{\star}=0.1822 M_{\odot}$.

disk of $13.7 \mathrm{Gyr}$. If we take $t_{\text {birth }}$ and $t_{\mathrm{MS}}$ as the time of birth and the main sequence time of the progenitor star, respectively, then the available time for the ELM WD to evolve during the WD stage is given by $13.7 \mathrm{Gyr}-t_{\mathrm{MS}}-t_{\mathrm{birth}}$. To obtain the maximum available time on the WD regime, and hence to find the lowest $T_{\text {eff }}$ that may be reached by a low-mass He WD, we set $t_{\text {birth }}=0$, that is, we assume that the progenitor was born at the beginning of star formation in the disk. In addition, the shortest ages for the possible progenitors of these stars must be considered. To assess this, we computed additional evolutionary calculations to estimate the maximum value of the initial mass of the progenitor stars at the ZAMS that may form ELM WDs, and we obtained a mass of $\sim 1.5 M_{\odot}\left(t_{\mathrm{MS}} \sim 1.5 \mathrm{Gyr}\right.$ for solar metallicity $)$. This is in line with the exploration made by Sun \& Arras (2018). Taking into account the $t_{\mathrm{MS}}$ of this progenitor, we explore the lowest $T_{\mathrm{eff}}$ values that can be reached by the $M_{\star}=0.1554 M_{\odot}$ and $M_{\star}=$ $0.1822 M_{\odot}$ non-flashing sequences, and the $M_{\star}=0.2025 M_{\odot}$ CNO-flashing sequence.

Results are illustrated in Fig. 3, in which these evolutionary sequences having canonical and thin $\mathrm{H}$ envelope are depicted in the $\log (g)-T_{\text {eff }}$ plane. We have also included in the figure the location of a sample of ELM WDs and also the known ELMVs (Hermes et al. 2012, 2013b,a; Kilic et al. 2015; Bell et al. 2015; Brown et al. 2016; Bell et al. 2017). The solid black line to the left connects points of equal age $(13.7 \mathrm{Gyr})$ for the canonical tracks, while the solid lines to the right connect points of equal age $(13.7 \mathrm{Gyr})$ but for the thin $\mathrm{H}$-envelope tracks. The dashed lines also correspond to the Galactic disk age limit, and are only marked as projections to delimit the region. The main result illustrated by Fig. 3 is the existence of a region named "Thin $\mathrm{H}$ envelope" in which only ELM WDs with thin $\mathrm{H}$ envelopes can be found. In fact, ELM WDs with canonical $\mathrm{H}$ envelopes are not expected to evolve to $T_{\text {eff }}$ values lower than the limit imposed by the left solid line. Only ELM WDs with thin H envelopes, and hence short cooling ages, are expected to evolve to such lower $T_{\text {eff }}$ (but not lower to the right solid line). More specifically, low-mass WDs with $M_{\star}<0.20 M_{\odot}$ with residual $\mathrm{H}$ burning (canonical envelopes) should be no cooler than roughly $7000 \mathrm{~K}$, as observations seem to show. But if such low-mass WDs were born with thinner $\mathrm{H}$ envelopes (for instance, due to unstable mass transfer) they could be expected at much lower effective temperatures (down to $\sim 2500 \mathrm{~K}$ ). This means that the detection of low-mass WDs in this region may indicate that such stars were formed with thin $\mathrm{H}$ envelopes. One possibility is through unstable mass loss via common-envelope episodes. In Fig. 3 we have also marked a zone named "Forbidden region" where ELM 
Table 1. Main characteristics $\left(T_{\text {eff }}, \log (g)\right.$ and $\left.t_{\text {cool }}\right)$ for some selected $0.1554,0.1822$ and $0.2025 M_{\odot}$ models with canonical and thin envelopes.

\begin{tabular}{|c|c|c|c|c|c|c|}
\hline$M_{\star}\left[M_{\odot}\right]$ & $T_{\text {eff }}[\mathrm{K}]$ & $\log (g)[\mathrm{cgs}]$ & $t_{\text {cool }}[\mathrm{Gyr}]$ & $T_{\text {eff }}[\mathrm{K}]$ & $\log (g)[\mathrm{cgs}]$ & $t_{\text {cool }}[\mathrm{Gyr}]$ \\
\hline & \multicolumn{3}{|c|}{ Thick envelope } & \multicolumn{3}{|c|}{ Thin envelope } \\
\hline \multirow[t]{15}{*}{0.1554} & 9420 & 5.682 & 2.077 & 9440 & 6.287 & 0.032 \\
\hline & 8930 & 5.858 & 2.811 & 8940 & 6.358 & 0.058 \\
\hline & 8420 & 5.995 & 3.633 & 8430 & 6.436 & 0.115 \\
\hline & 7930 & 6.108 & 4.742 & 7930 & 6.505 & 0.203 \\
\hline & 7430 & 6.245 & 10.12 & 7430 & 6.561 & 0.309 \\
\hline & & & & 6930 & 6.611 & 0.444 \\
\hline & & & & 6430 & 6.654 & 0.608 \\
\hline & & & & 5930 & 6.694 & 0.820 \\
\hline & & & & 5430 & 6.737 & 1.112 \\
\hline & & & & 4930 & 6.812 & 1.593 \\
\hline & & & & 4430 & 6.834 & 2.145 \\
\hline & & & & 3930 & 6.855 & 2.968 \\
\hline & & & & 3430 & 6.875 & 4.356 \\
\hline & & & & 2930 & 6.912 & 8.469 \\
\hline & & & & 2540 & 6.927 & 12.21 \\
\hline \multirow[t]{17}{*}{0.1822} & 10430 & 6.479 & 3.583 & 10420 & 6.548 & 0.052 \\
\hline & 9930 & 6.516 & 4.249 & 9930 & 6.595 & 0.087 \\
\hline & 9430 & 6.554 & 5.066 & 9430 & 6.642 & 0.139 \\
\hline & 8930 & 6.590 & 6.074 & 8940 & 6.684 & 0.203 \\
\hline & 8420 & 6.624 & 7.203 & 8440 & 6.722 & 0.279 \\
\hline & 7940 & 6.655 & 8.848 & 7930 & 6.756 & 0.374 \\
\hline & 7430 & 6.685 & 10.69 & 7440 & 6.787 & 0.486 \\
\hline & 6930 & 6.713 & 13.03 & 6920 & 6.816 & 0.631 \\
\hline & & & & 6440 & 6.844 & 0.808 \\
\hline & & & & 5930 & 6.872 & 1.046 \\
\hline & & & & 5430 & 6.904 & 1.402 \\
\hline & & & & 4930 & 6.967 & 1.971 \\
\hline & & & & 4420 & 6.986 & 2.682 \\
\hline & & & & 3930 & 7.008 & 4.004 \\
\hline & & & & 3430 & 7.021 & 5.675 \\
\hline & & & & 2930 & 7.039 & 9.497 \\
\hline & & & & 2550 & 7.046 & 12.18 \\
\hline \multirow[t]{17}{*}{0.2025} & 10430 & 6.695 & 0.003 & 10430 & 6.798 & 0.004 \\
\hline & 9910 & 6.706 & 0.059 & 9940 & 6.809 & 0.007 \\
\hline & 9420 & 6.719 & 0.121 & 9440 & 6.825 & 0.014 \\
\hline & 8950 & 6.735 & 0.188 & 8940 & 6.844 & 0.034 \\
\hline & 8430 & 6.753 & 0.278 & 8450 & 6.868 & 0.093 \\
\hline & 7930 & 6.771 & 0.380 & 7910 & 6.895 & 0.199 \\
\hline & 7430 & 6.790 & 0.499 & 7450 & 6.916 & 0.314 \\
\hline & 6930 & 6.811 & 0.650 & 6960 & 6.937 & 0.463 \\
\hline & 6440 & 6.833 & 0.849 & 6430 & 6.960 & 0.673 \\
\hline & 5930 & 6.859 & 1.117 & 5930 & 6.981 & 0.924 \\
\hline & 5430 & 6.889 & 1.480 & 5430 & 7.007 & 1.284 \\
\hline & 4930 & 6.941 & 2.031 & 4930 & 7.058 & 1.886 \\
\hline & 4430 & 6.998 & 2.844 & 4420 & 7.075 & 2.729 \\
\hline & 3930 & 7.031 & 4.418 & 3930 & 7.093 & 4.132 \\
\hline & 3430 & 7.047 & 6.166 & 3430 & 7.113 & 6.810 \\
\hline & 2930 & 7.058 & 8.525 & 2940 & 7.120 & 9.502 \\
\hline & 2530 & 7.064 & 11.17 & 2530 & 7.124 & 12.13 \\
\hline
\end{tabular}

WDs are not expected to be found because to reach such region, evolutionary times larger than the Galactic disk age would be needed (even assuming ELM WDs with thin $\mathrm{H}$ envelopes).

Another observable consequence of a reduction of the $\mathrm{H}$ envelope concerns its impact on the surface gravity. It is clear from Fig. 3 and also from Table 1 that reducing the thickness of the $\mathrm{H}$ envelope yields evolutionary sequences with substantially higher gravity values. This is because a reduction in the $\mathrm{H}$ content causes the envelope to become denser, thus implying a decrease in the stellar radius. At variance with averagemass WDs, where the mass of residual $\mathrm{H}$ is much smaller, the impact on the gravity becomes more significant in low-mass Hecore WDs. The increase in gravity resulting from a reduction of the $\mathrm{H}$ envelope has consequences for the spectroscopic 


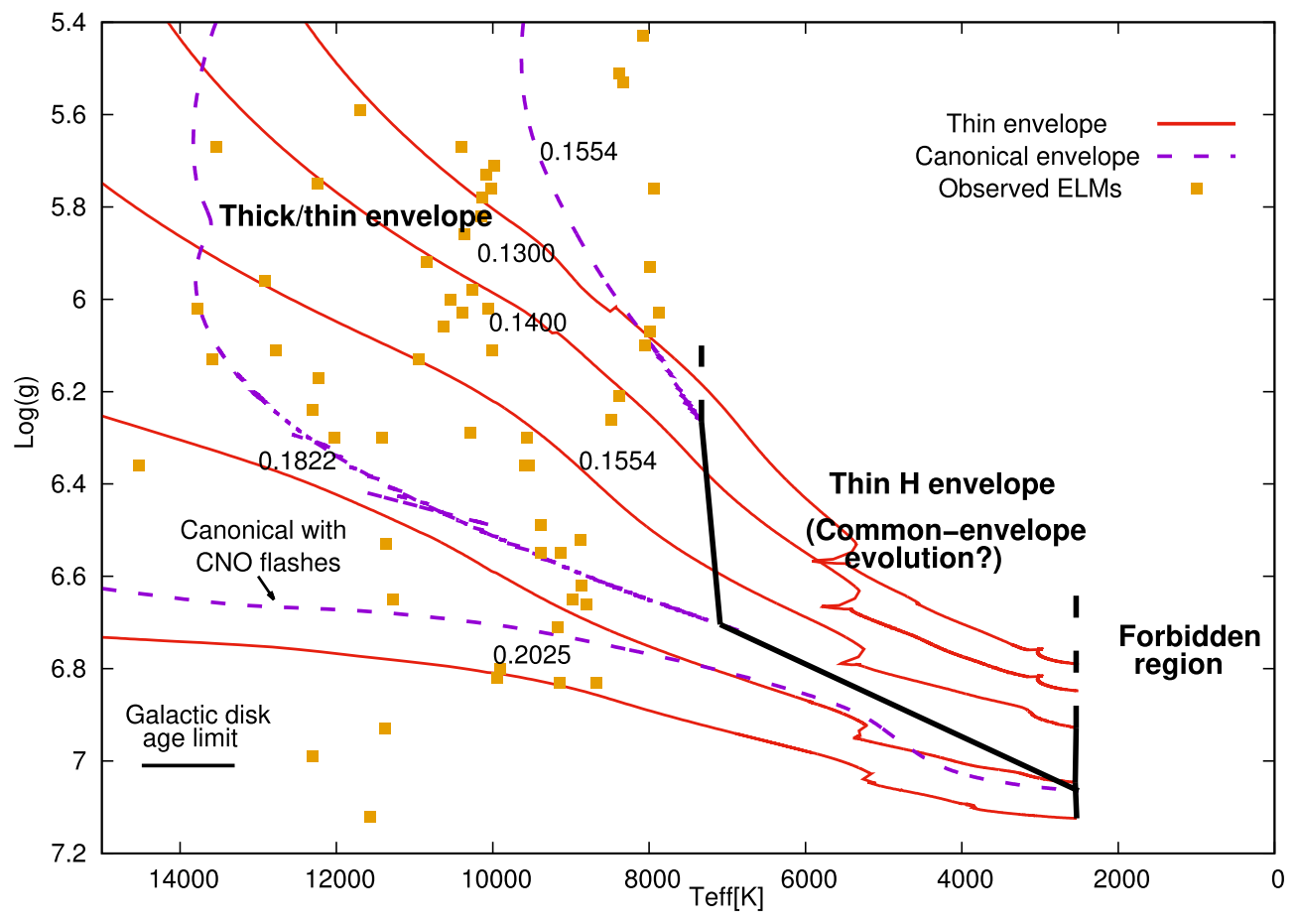

Fig. 3. Evolutionary sequences of lowmass WDs with different thicknesses of the $\mathrm{H}$ envelope (canonical and thin) in the $\log (g)$ vs. $T_{\text {eff }}$ plane. The vertical black line to the left (right) connects points of equal age, $13.7 \mathrm{Gyr}$, for the canonical (thin $\mathrm{H}$ envelopes) tracks. The dashed lines also correspond to the galactic disk age limit, and are only marked as projections to delimit the region. We have also included the 0.130 and $0.140 M_{\odot}$ artificial evolutionary sequences. Also included are the observed ELMs and ELMVs. The progenitor considered has $M_{\star}=1.5 M_{\odot}$. determination of the star parameters. At first glance, the uncertainty in the stellar mass due to the uncertainty in the thickness of the $\mathrm{H}$ envelope is of at least $\sim 0.025 M_{\odot}$.

The above-mentioned expectations are based on the asssumption that the progenitor star has the maximum value of the initial mass, and hence the shortest ages, to form ELM WDs, that is, $1.5 M_{\odot}$. Now we explore how the conclusions are altered if the mass of the progenitor star is changed. In particular, results for ELM WDs coming from 1.0 and $1.3 M_{\odot}$ progenitor stars are shown in Fig. 4. We note that lines of equal ages shift now to higher $T_{\text {eff }}$ values in comparison with the lines predicted in the case of the $1.5 M_{\odot}$ progenitor, as is expected because of the larger progenitor ages. However, this shift is not significant and we can conclude that these boundaries are not very sensitive to the mass of the progenitor. Specifically, if low-mass WDs with $M_{\star}<0.18 M_{\odot}$ were formed with thick $\mathrm{H}$ envelopes and came from a progenitor with $1.3 M_{\odot}$, they would not be likely to be cooler than $\sim 7400 \mathrm{~K}$, and if such low-mass WDs were born with thin $\mathrm{H}$ envelopes, they could reach down to $\sim 2700 \mathrm{~K}$. For the $1.0 M_{\odot}$ progenitor, the limits are roughly $8100 \mathrm{~K}$ and $3500 \mathrm{~K}$, for the thick and thin $\mathrm{H}$ envelope, respectively. The region where only thin $\mathrm{H}$ envelope can be found remains mostly unchanged and well defined.

Finally, we note that all of our thin $\mathrm{H}$ envelope sequences experience a sort of hook in their track between $T_{\text {eff }} \sim 5000$ and $6000 \mathrm{~K}$. The reason for this is related to the deepening of the convection zone that reaches the $\mathrm{H} / \mathrm{He}$ transition region, enriching the envelope with $\mathrm{He}$ and thereby making the star more compact.

\section{Summary and conclusions}

Here we present new evolutionary sequences of ELM WDs with thin $\mathrm{H}$ envelopes and study the differences in the cooling times comparing thin and thick H-envelope sequences. ELM WD stars are thought to be the result of strong mass-loss episodes in interactive binary systems before the He flash during the RGB-phase of low-mass stars (Sarna et al. 2000; Althaus et al. 2013; Istrate et al. 2016). It is currently accepted that these stars are formed either through unstable mass loss, via common-envelope episodes, for example, or stable mass loss via Roche-lobe overflow in close binary systems (for a recent discussion see Istrate et al. 2016). All of the existing studies (e.g., Althaus et al. 2013; Istrate et al. 2016) are performed considering ELM WDs evolving from progenitors that have experienced stable mass transfer, leading to thick $\mathrm{H}$ envelopes and hence, to very long cooling ages due to residual $\mathrm{H}$ burning. However, we cannot rule out a scenario where these stars are formed through unstable mass loss, leading to ELM WDs with thin $\mathrm{H}$ envelopes unable to sustain residual $\mathrm{H}$ burning, resulting in much shorter cooling ages.

In the present paper, we determine the lowest $T_{\text {eff }}$ that could be reached by ELM WDs. We studied the cooling times of ELM WD models with canonical (thick) $\mathrm{H}$ envelopes taken from Althaus et al. (2013), and also ELM WD models characterized by thin $\mathrm{H}$ envelopes. This last set of models was generated on the basis of models with thick envelopes, for which we artificially reduced the thickness of the $\mathrm{H}$ envelope at high luminosities. We stress that the reduction in the thickness of the $\mathrm{H}$ envelope does not need to be large for the nuclear burning to become negligible: for instance, for the sequence with $0.1554 M_{\odot}$, only a factor of reduction of $\sim 2.4$ is enough. We analyzed the cooling ages of our WD sequences considering the Galactic disk age, and assumed that stars were born at the same time as the formation of the disk, meaning they would have had enough time to reach the minimum $T_{\text {eff }}$. We also considered the age of some possible progenitors that can form ELM WDs. We found that there is a strictly limited region in the $\log (g)$ versus $T_{\text {eff }}$ plane where only ELM WD stars with thin $\mathrm{H}$ envelopes can be found. We also found that changing the progenitor mass does not significantly change the lowest $T_{\text {eff }} \mathrm{s}$ reached by these stars. Additionally, we found that a reduction of the $\mathrm{H}$ envelope has an important observational impact, because it increases the value of $g$, leading to consequences in the spectroscopic determinations for these stars.

Our results show that it would be possible to find ELM WD stars at very low $T_{\text {eff }}$ if they had a thin $\mathrm{H}$ envelope. This being the case, we could conclude that these stars experienced unstable mass loss during their previous evolution, shedding light on 


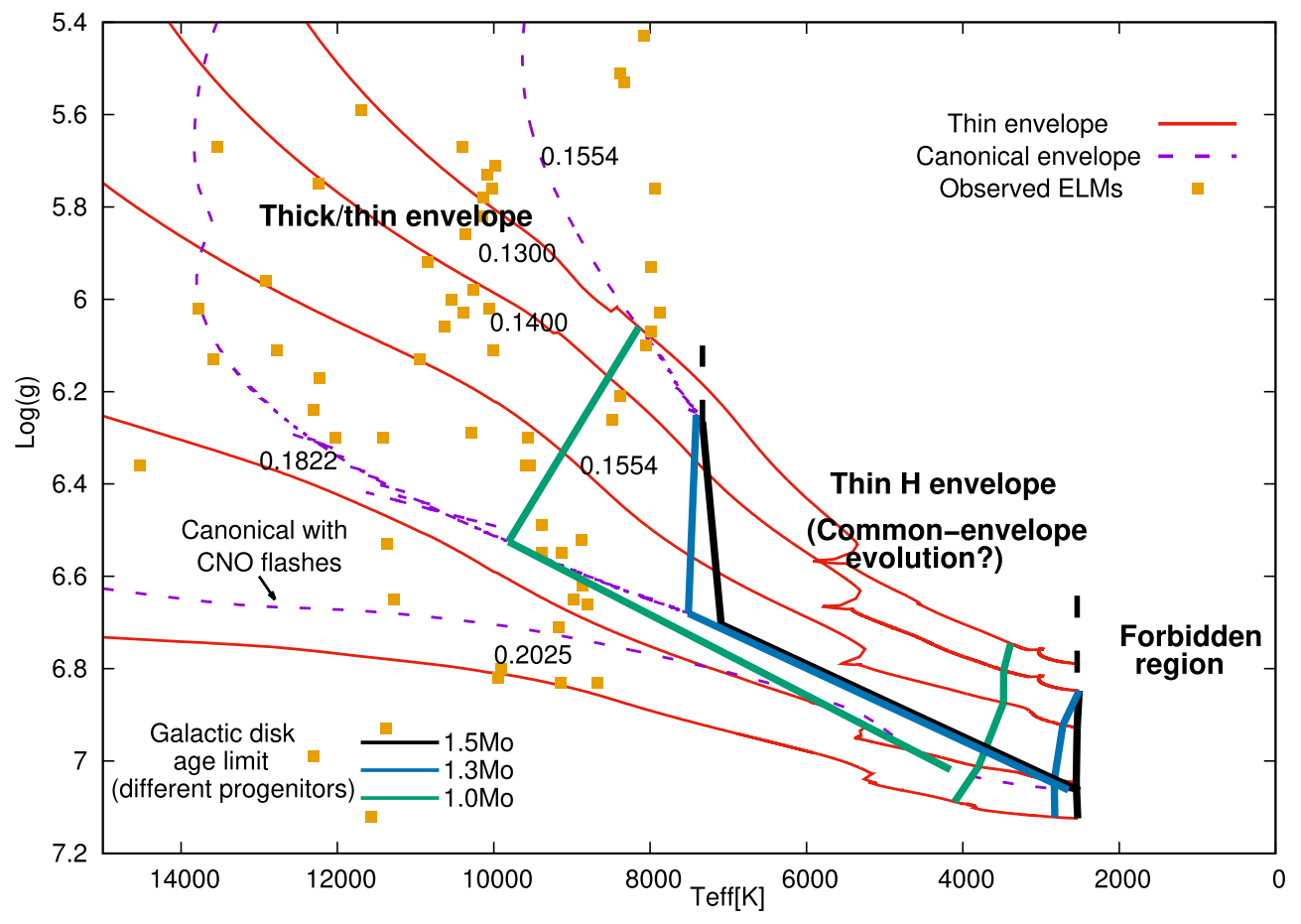

Fig. 4. As in Fig. 3 but for different masses of the progenitor star (1.0, 1.3 and $\left.1.5 M_{\odot}\right)$, marked with different solid colored lines. the formation of such stars. We are aware that the detection of cool ELM WDs is a difficult task, due to the inaccuracy of the spectroscopic technique below $7000 \mathrm{~K}$. However, hopefully with Gaia parallax measurements, we might be able to identify such cool ELM WDs.

Acknowledgements. We wish to thank our anonymous referee for the constructive comments and suggestions that greatly improved the original version of the paper. We also wish to warmly thank the helpful comments of M. Kilic, W. R. Brown and K. J. Bell. Part of this work was supported by AGENCIA through the Programa de Modernización Tecnológica BID 1728/OC-AR, and by the PIP 112-200801-00940 grant from CONICET. This research made use of NASA Astrophysics Data System.

\section{References}

Althaus, L. G., Serenelli, A. M., \& Benvenuto, O. G. 2001, MNRAS, 323, 471

Althaus, L. G., Serenelli, A. M., Panei, J. A., et al. 2005, A\&A, 435, 631

Althaus, L. G., Panei, J. A., Romero, A. D., et al. 2009, A\&A, 502, 207

Althaus, L. G., Córsico, A. H., Isern, J., \& García-Berro, E. 2010, A\&ARv, 18 471

Althaus, L. G., Miller Bertolami, M. M., \& Córsico, A. H. 2013, A\&A, 557, A19

Althaus, L. G., Camisassa, M. E., Miller Bertolami, M. M., Córsico, A. H., \& García-Berro, E. 2015, A\&A, 576, A9

Bell, K. J., Kepler, S. O., Montgomery, M. H., et al. 2015, in 19th European Workshop on White Dwarfs, eds. P. Dufour, P. Bergeron, \& G. Fontaine, ASP Conf. Ser., 493, 217

Bell, K. J., Gianninas, A., Hermes, J. J., et al. 2017, ApJ, 835, 180

Bono, G., Salaris, M., \& Gilmozzi, R. 2013, A\&A, 549, A102

Brown, W. R., Kilic, M., Allende Prieto, C., \& Kenyon, S. J. 2010, ApJ, 723, 1072

Brown, W. R., Gianninas, A., Kilic, M., Kenyon, S. J., \& Allende Prieto C. 2016, ApJ, 818, 155

Burgers, J. M. 1969, Flow Equations for Composite Gases (New York: Academic Press)
Cassisi, S., Potekhin, A. Y., Pietrinferni, A., Catelan, M., \& Salaris, M. 2007. ApJ, 661, 1094

Clayton, M., Podsiadlowski, P., Ivanova, N., \& Justham, S. 2017, MNRAS, 470, 1788

De Marco, O., \& Soker, N. 2002, PASP, 114, 602

Ergma, E., Sarna, M. J., \& Gerkevit-Antipova, J. 2001, MNRAS, 321, 71

Fontaine, G., \& Brassard, P. 2008, PASP, 120, 1043

García-Berro, E., Torres, S., Althaus, L. G., et al. 2010, Nature, 465, 194

Gianninas, A., Kilic, M., Brown, W. R., Canton, P., \& Kenyon, S. J. 2015, ApJ, 812,167

Haft, M., Raffelt, G., \& Weiss, A. 1994, ApJ, 425, 222

Hansen, B. M. S., Kalirai, J. S., Anderson, J., et al. 2013, Nature, 500, 51

Hermes, J. J., Montgomery, M. H., Winget, D. E., et al. 2012, ApJ, 750, L28

Hermes, J. J., Montgomery, M. H., Gianninas, A., et al. 2013a, MNRAS, 436, 3573

Hermes, J. J., Montgomery, M. H., Winget, D. E., et al. 2013b, ApJ, 765, 102

Iglesias, C. A., \& Rogers, F. J. 1996, ApJ, 464, 943

Istrate, A. G., Marchant, P., Tauris, T. M., et al. 2016, A\&A, 595, A35

Itoh, N., Hayashi, H., Nishikawa, A., \& Kohyama, Y. 1996, ApJS, 102, 411

Ivanova, N., \& Nandez, J. L. A. 2016, MNRAS, 462, 362

Jeffery, E. J., von Hippel, T., DeGennaro, S., et al. 2011, ApJ, 730, 35

Kilic, M., Hermes, J. J., Gianninas, A., \& Brown, W. R. 2015, MNRAS, 446, L26

Koester, D., Voss, B., Napiwotzki, R., et al. 2009, A\&A, 505, 441

Magni, G., \& Mazzitelli, I. 1979, A\&A, 72, 134

Maxted, P. F. L., Anderson, D. R., Burleigh, M. R., et al. 2011, MNRAS, 418, 1156

Nandez, J. L. A., \& Ivanova, N. 2016, MNRAS, 460, 3992

Nelemans, G., \& Tauris, T. M. 1998, A\&A, 335, L85

Sabach, E., \& Soker, N., 2018, MNRAS, 473, 286

Sarna, M. J., Ergma, E., \& Gerkevit-Antipova, J. 2000, MNRAS, 316, 84

Strickler, R. R., Cool, A. M., Anderson, J., et al. 2009, ApJ, 699, 40

Sun, M., \& Arras, P. 2018, ApJ, 858, 14

Tassoul, M., Fontaine, G., \& Winget, D. E. 1990, ApJS, 72, 335

Torres, S., García-Berro, E., Althaus, L. G., \& Camisassa, M. E. 2015, A\&A, 581, A90

van den Heuvel, E. P. J., \& van Paradijs J. 1988, Nature, 334, 227

Winget, D. E., \& Kepler, S. O. 2008, ARA\&A, 46, 157

Winget, D. E., Kepler, S. O., Campos, F., et al. 2009, ApJ, 693, L6 